\title{
Robot Localization using Uncalibrated Camera Invariants *
}

\author{
Michael Werman $^{\dagger} \quad$ Subhashis Banerjee秦 Sumantra Dutta Roy ${ }^{\ddagger} \quad$ MaoLin Qiu $^{\dagger}$
}

\begin{abstract}
We describe a set of image measurements which are invariant to the camera internals but are location variant. We show that using these measurements it is possible to calculate the self-localization of a robot using known landmarks and uncalibrated cameras. We also show that it is possible to compute, using uncalibrated cameras, the Euclidean structure of $3 D$ world points using multiple views from known positions. We are free to alter the internal parameters of the camera during these operations. Our initial experiments demonstrate the applicability of the method.
\end{abstract}

\section{Introduction}

Improving robot efficiency in advanced industrial automation requires the inclusion of sensory information at many levels, one of the most important is online task control. Range finders, proximity sensors or wrist force sensors can only address the sensing problem in a reliable way when the robot is already at its target. Visual sensors can be used on-line using the visual servoing approach. This technique, which consists of realizing an image-based feedback control loop is of increasing popularity. However, generally speaking, it is currently based on the use of a single camera and operates only on the premise that all the components are fully calibrated: camera, robot, and world. The related calibration procedures $[15,5]$ are tedious, nonflexible and expensive. Mobile robot navigation and docking require the robot to know where it is. This is usually achieved through some combination of odometry and landmark observations. The possibility of using visual landmarks viewed through uncalibrated cameras for self-localization will reduce the cost and enlarge the potential market of a robot system.

Our main objective is to remove the calibration bottleneck in visually-guided robots. We address the problem of accurately positioning a robot, with up to 6 degrees of freedom, using only pictorial information from

\footnotetext{
* Supported by Israel - India Scientific Cooperation 8970-1-97 and Espirit project 26247 - Vigor

†Institute of Computer Science, Hebrew University, 91904 Jerusalem, Israel. Email: \{werman,qiuml\}@cs.huji.ac.il

$\ddagger$ Department of Computer Science and Engineering, Indian Institute of Technology, New Delhi 110016, India. Email: $\{$ suban,sumantra\}@cse.iitd.ernet.in
}

an uncalibrated camera. We are interested in localization even in the absence of an a priori pose estimate, obviating the need of using Kalman filtering or optical flow techniques to estimate odometry. We present a new method for self-localization based on image measurements which are invariant to the internal parameters of the camera. The invariant measures depend only on (i) the external parameters of the camera which relate the camera and world coordinate systems through rotations and translations, and (ii) the Euclidean coordinates (structure) of the landmarks. Given the landmarks, we can compute the external parameters of the camera from the image measurements. Conversely, given the robot position in a world coordinate system and the point correspondences in multiple views, we can compute the 3D Euclidean structure of the points.

The problem of landmark-based position recognition was first formalized by Sugihara [12] as a computational geometry problem, assuming a completely calibrated camera. Recently, Quan and Lan [10] addressed the problem of finding linear algorithms for pose determination using calibrated cameras and known landmarks. Many robot localization methods rely on landmarks which are either artificially added to the environment or based on strong assumptions with respect to the environment [1, 8, 13]. For example, Krotkov [7] relies on the assumption that the environment is structured in such a way that vertical lines can be easily extracted as landmarks. This assumption is problematic in two ways. First, it places a restriction on the kinds of environments that can be explored, and second, it places a restriction on the pose of the camera. Basri and Rivlin [2] have also exploited the geometric behavior of landmarks in selected model images to provide navigation information. Exploiting an assumption of global invertibility of the imaging function and it being an affine projection, Nayar [9] has shown that subspace methods can provide accurate positional feedback in sufficiently constrained environments. A key assumption in his work is that each possible viewing position gives rise to a unique image. In a similar work, Thrun [14] derives a probabilistic approach to obtain a pose estimate using a neural net. In the works of both $\mathrm{Na}^{-}$ yar and Thrun, however, all significant variations in the set of possible images, including those due to lighting 
variations, must be explicitly sampled and encoded. In other work, it has been shown that localization can be achieved despite unanticipated illumination variations. That method can also deal with non-invertibility of the imaging transform, a problem that is typical in unconstrained environments.

Our approach is close to that of Dudek et al. [3, 11] which uses image landmarks to perform position estimation, but learns these landmarks from a preliminary traversal of the environment (i.e. an off-line mapping phase). They have to use the same camera on subsequent localization tasks as they do not compute an invariant but just use the feature image.

\section{Image invariants with respect to camera internals}

Consider the case when the image projection $\mathbf{m}^{T}=$ $(u, v, 1)^{t}$ of a world point $\mathbf{P}^{T}=(X, Y, Z, 1)^{t}$ is described by the following model of imaging [5]

$$
\lambda \mathbf{m}=\mathbf{A}[\mathbf{R} \mid \mathbf{t}] \mathbf{P}
$$

where $\mathbf{A}$ is the matrix of camera internals given by

$$
\mathbf{A}=\left[\begin{array}{ccc}
f_{x} & 0 & u_{0} \\
0 & f_{y} & v_{0} \\
0 & 0 & 1
\end{array}\right]
$$

$\mathbf{R}$ and $\mathbf{t}$ are the rotation and the translation between the world and the camera frames, and $[\mathbf{R} \mid \mathbf{t}]$ is the matrix of camera externals given by

$$
[\mathbf{R} \mid \mathbf{t}]=\left[\begin{array}{llll}
r_{11} & r_{12} & r_{13} & t_{1} \\
r_{21} & r_{22} & r_{23} & t_{2} \\
r_{31} & r_{32} & r_{33} & t_{3}
\end{array}\right]=\left[\begin{array}{c}
\mathbf{r}_{1} \\
\mathbf{r}_{2} \\
\mathbf{r}_{3}
\end{array}\right]
$$

Rewriting Equation 1, we have:

$$
\begin{aligned}
& u=f_{x} \frac{\mathbf{r}_{1} \mathbf{P}}{\mathbf{r}_{3} \mathbf{P}}+u_{0} \\
& v=f_{y} \frac{\mathbf{r}_{2} \mathbf{P}}{\mathbf{r}_{3} \mathbf{P}}+v_{0}
\end{aligned}
$$

Suppose we know three $3 \mathrm{D}$ points, $\mathbf{P}_{p}^{T}=$ $\left(X_{p}, Y_{p}, Z_{p}, 1\right)^{T}, p \in\{i, j, k\}$, and their images on the image plane, $\mathbf{m}_{p}^{T}=\left(u_{p}, v_{p}, 1\right)^{T}, p \in\{i, j, k\}$, then by eliminating the internals of the camera, we obtain

$$
\begin{aligned}
& K_{i j k}=\frac{u_{i}-u_{j}}{u_{i}-u_{k}}=\frac{\frac{\mathbf{r}_{1} \mathbf{P}_{i}}{\mathbf{r}_{3} \mathbf{P}_{i}}-\frac{\mathbf{r}_{1} \mathbf{P}_{j}}{\mathbf{r}_{3} \mathbf{P}_{j}}}{\frac{\mathbf{r}_{1} \mathbf{P}_{i}}{\mathbf{r}_{3} \mathbf{P}_{i}}-\frac{\mathbf{r}_{1} \mathbf{P}_{k}}{\mathbf{r}_{3} \mathbf{P}_{k}}} \\
& J_{i j k}=\frac{v_{i}-v_{j}}{v_{i}-v_{k}}=\frac{\frac{\mathbf{r}_{2} \mathbf{P}_{i}}{\mathbf{r}_{3} \mathbf{P}_{i}}-\frac{\mathbf{r}_{2} \mathbf{P}_{j}}{\mathbf{r}_{3} \mathbf{P}_{j}}}{\frac{\mathbf{r}_{2} \mathbf{P}_{i}}{\mathbf{r}_{3} \mathbf{P}_{i}}-\frac{\mathbf{r}_{2} \mathbf{P}_{k}}{\mathbf{r}_{3} \mathbf{P}_{k}}},
\end{aligned}
$$

in which $K_{i j k}$ and $J_{i j k}$ are image measurements that are functions of $\mathbf{R}, \mathbf{t}$ and $\mathbf{P}_{p}, p \in\{i, j, k\}$, and are independent of the internals of the camera. Thus the above equations can be re-written as the following constraints

$$
\begin{aligned}
& K_{i j k}=f_{i j k}\left(\mathbf{R}, \mathbf{t}, \mathbf{P}_{i}, \mathbf{P}_{j}, \mathbf{P}_{k}\right) \\
& J_{i j k}=g_{i j k}\left(\mathbf{R}, \mathbf{t}, \mathbf{P}_{i}, \mathbf{P}_{j}, \mathbf{P}_{k}\right)
\end{aligned}
$$

The left hand sides of Equation 6 represent image measurement based on three points which are invariant to the camera internals. The right hand sides are nonlinear trigonometric expressions which are functions of only the camera externals and the structure (Euclidean coordinates) of the three points.

Given $n \geq 3$ control points in the $3 \mathrm{D}$ world, we get $2(n-2)$ independent constraints from one view of the $3 \mathrm{D}$ scene. Suppose the number of views is $N$, then the total number of the independent constraints is $2 N(n-2)$.

\subsection{Pose estimation using Euclidean land- marks}

Now suppose that we know the Euclidean coordinates $\left(X_{i}, Y_{i}, Z_{i}\right)$ of 5 points in the world coordinate system. Six independent invariant measurements give us six equations in terms of the six unknowns in $(\mathbf{R}, \mathbf{t})$. The six equations can be solved numerically for a complete pose estimation using an uncalibrated camera and known landmarks.

In the case of constrained planar motion, $\mathbf{R}$ has only one degree of freedom and $\mathbf{t}$ has two degrees of freedom. The total number of unknowns in such a case is three. Then four control points are sufficient for pose estimation.

It turns out that in some special cases it is possible to obtain closed-form or linear solutions to the pose estimation problem using the image invariants. We will discuss some of these special cases in Section 3 .

\subsection{D Euclidean reconstruction from known ego-motions}

Conversely, suppose that we have a robot whose ego-motion and odometry can be known exactly from positional encoders and we know the $\mathbf{R}$ and $\mathbf{t}$ at $N$ locations of the camera. With $n$ control points we have $3 n$ unknowns. In order to compute the Euclidean coordinates of these $3 \mathrm{D}$ points, the relationship $2 N(n-2) \geq 3 n$ must be satisfied. Thus with 3 views $(N=3)$ of at least 4 points $(n \geq 4)$ we can compute the $3 \mathrm{D}$ Euclidean structure provided the pose of each of the camera stations are known. Some possibilities of number of views $N$ and number of control points $n$ are $N=3$ and $n \geq 4 ; N=5$ and $n \geq 3$.

We prove in the appendix that 3 views are mandatory for 3D Euclidean reconstruction from known ego- 
motions in the case that the internals of the camera change across views.

\subsection{Interpolation of camera motion}

In the case discussed in Section 2.2, suppose we do not know the $\mathbf{R}$ and $\mathbf{t}$ at one of the $N$ locations of the camera. Then we have the relationship $2 N(n-2) \geq$ $(3 n+6)$. Thus with 4 views of at least 5 points we can compute all the unknowns. If the $3 \mathrm{D}$ structure is not of interest then the coordinates of the $3 \mathrm{D}$ control points can be eliminated from the constraints and we can arrive at the equations for interpolation of camera motion. Some possibilities for $N$ and $n$ are $N=4$ and $n \geq 5 ; N=5$ and $n \geq 4 ; N=8$ and $n \geq 3$.

\subsection{Interpolation of the image measure- ments}

Suppose we know the $\mathbf{R}$ and $\mathbf{t}$ for all stations but do not know the image measurements $K$ 's and $J$ 's corresponding to one of the stations. Now we have the relationship $2 N(n-2) \geq 3 n+2(n-2)$. Thus we can interpolate the image measurements with any of the following situations - i) $N=3$ and $n \geq 8$ ii) $N=4$ and $n \geq 4$ iii) $N=6$ and $n \geq 3$. Like in the previous case we can eliminate the coordinates of the $3 \mathrm{D}$ control points if the Euclidean structure is not of interest.

In each of the above cases it is possible to use a larger number of control points to robustly estimate the parameters using a suitable nonlinear optimization technique.

\section{Special cases}

In what follows we discuss some of the special cases.

\subsection{Rotation only about Z-axis}

Let $\mathbf{X}_{w} \mathbf{Y}_{w} \mathbf{Z}_{w}$ be the world coordinate system. Consider restricted motion with rotation $\theta$ only about the $\mathbf{Z}_{w^{-}}$axis. This would be the case of a robot moving on the ground with a special camera looking vertically up at the landmarks on the ceiling to facilitate localization.

Suppose there are three 3D control points lying on the $\mathbf{X}_{w} \mathbf{Y}_{w}$ plane (ceiling) of the 3D world coordinate system, denoted as $\left(X_{i}, Y_{i}, 0,1\right),\left(X_{j}, Y_{j}, 0,1\right)$ and $\left(X_{k}, Y_{k}, 0,1\right)$, with the corresponding image coordinates being $\left(u_{i}, v_{i}, 1\right),\left(u_{j}, v_{j}, 1\right)$ and $\left(u_{k}, v_{k}, 1\right)$, respectively. The image projection is

$$
\begin{aligned}
& u_{p}=f_{x} \frac{X_{p} \cos \theta+Y_{p} \sin \theta+t_{1}}{t_{3}}+u_{0} \\
& v_{p}=f_{y} \frac{-X_{p} \sin \theta+Y_{p} \cos \theta+t_{2}}{t_{3}}+v_{0}
\end{aligned}, p \in\{i, j, k\} .
$$

By eliminating the internals of the camera from Equation 7 , we obtain

$$
\begin{aligned}
& \frac{u_{i}-u_{j}}{u_{i}-u_{k}}=\frac{\left(X_{i}-X_{j}\right) \cos \theta+\left(Y_{i}-Y_{j}\right) \sin \theta}{\left(X_{i}-X_{k}\right) \cos \theta+\left(Y_{i}-Y_{k}\right) \sin \theta} \\
& \frac{v_{i}-v_{j}}{v_{i}-v_{k}}=\frac{\left(-X_{i}+X_{j}\right) \sin \theta+\left(Y_{i}-Y_{j}\right) \cos \theta}{\left(-X_{i}+X_{k}\right) \sin \theta+\left(Y_{i}-Y_{k}\right) \cos \theta}
\end{aligned}
$$

Let $K=\left(u_{i}-u_{j}\right) /\left(u_{i}-u_{k}\right)$ and $J=\left(v_{i}-v_{j}\right) /\left(v_{i}-v_{k}\right)$. Let $\mathcal{P}_{p q}$ stand for $\mathcal{P}_{p}-\mathcal{P}_{q}$. We obtain

$$
\tan \theta=\frac{-K X_{i k}+X_{i j}}{K Y_{i k}-Y_{i j}}=\frac{-J Y_{i k}+Y_{i j}}{J X_{k i}-X_{j i}} .
$$

We can easily compute $\theta$ (the rotation about $\mathbf{Z}$ ) from Equation 9. Once $\theta$ is obtained, the translation vector $\mathbf{t}$ can be computed as follows. Suppose we get another $3 \mathrm{D}$ control point which is not on the plane $\mathbf{X}_{w} \mathbf{Y}_{w}$, say $\left(X_{j}, Y_{j}, Z_{j}, 1\right)$ (let us not stop at just painting the ceiling, but hang a few sticks as well). We compute $K$ and $J$ the in same way as in Equation 8 . Then we have

$$
\begin{aligned}
\frac{t_{3}}{Z_{j}} & =\frac{X_{i} \cos \theta+Y_{i} \sin \theta+t_{1}+K_{i k}}{K_{i k}+X_{j} \cos \theta+Y_{j} \sin \theta-\left(X_{i} \cos \theta+Y_{i} \sin \theta\right)} \\
\frac{t_{3}}{Z_{j}} & =\frac{-X_{i} \sin \theta+Y_{i} \cos \theta+t_{2}+J_{i k}}{J_{i k}-X_{j} \sin \theta+Y_{j} \cos \theta-\left(-X_{i} \sin \theta+Y_{i} \cos \theta\right)}
\end{aligned}
$$

where $K_{i k}=K\left(X_{i} \cos \theta+Y_{i} \sin \theta-\left(X_{k} \cos \theta+Y_{k} \sin \theta\right)\right)=$ $K K_{0}$; and $J_{i k}=J\left(-X_{i} \sin \theta+Y_{i} \cos \theta-\left(-X_{k} \sin \theta+\right.\right.$ $\left.\left.Y_{k} \cos \theta\right)\right)=J J_{0}$. By equating the two equations in 10 , and rearranging the terms, we arrive at

$$
\left(b_{j}-d_{j}\right) t_{1}+\left(c_{j}-a_{j}\right) t_{2}=a_{j} d_{j}-c_{j} b_{j}
$$

where $a_{j}=X_{j} \cos \theta+Y_{j} \sin \theta ; b_{j}=-X_{j} \sin \theta+Y_{j} \cos \theta$; $c_{j}=X_{i} \cos \theta+Y_{i} \sin \theta-K_{i k} ;$ and $d_{j}=-X_{i} \sin \theta+$ $Y_{i} \cos \theta-J_{i k}$. If we know more $3 \mathrm{D}$ control points which are not on the $\mathbf{X}_{w} \mathbf{Y}_{w}$ plane, we can get more equations in the form of Equation 11. In such a case, a linear least squares technique can be used to solve for $t_{1}$ and $t_{2}$. Further, $t_{3}$ can be computed using the following relationship

$$
t_{3}=Z_{j} \frac{c_{j}+t_{1}}{a_{j}-c_{j}}=Z_{j} \frac{d_{j}+t 2}{b_{j}-d_{j}}
$$

Thus, in this special case, we can linearly solve the localization problem given three Euclidean landmarks on the $\mathbf{X}_{w} \mathbf{Y}_{w}$ plane and at least one landmark off the $\mathbf{X}_{w} \mathbf{Y}_{w}$ plane.

\subsection{Motion and Struct ure from planar mo- tion and rotation about $\mathrm{Z}$}

In the case described above, suppose we do not know the coordinates of the control points on the $\mathbf{X}_{w} \mathbf{Y}_{w}$ plane (the ceiling). Consider planar motion on a plane parallel to the $\mathbf{X}_{w} \mathbf{Y}_{w}$ plane and rotation only about $\mathbf{Z}_{w}$. By rearranging the terms in Equation 8 and letting $\mathcal{P}_{p q}$ stand for $\mathcal{P}_{p}-\mathcal{P}_{q}$, we obtain

$$
\begin{aligned}
& X_{i j}+Y_{i j} \tan \theta-K_{i j k} X_{i k}-K_{i j k} \tan \theta Y_{i k}=0 \\
& X_{i j} \tan \theta-Y_{i j}-J_{i j k} \tan \theta X_{i k}+J_{i j k} Y_{i k}=0
\end{aligned}
$$

Now suppose we know the image points of any $43 \mathrm{D}$ points on the $\mathbf{X}_{w} \mathbf{Y}_{w}$ plane, which are in general position. We then have totally $2(4-2)=4$ equations as follows

$$
\mathbf{M}(\theta) \mathbf{X}=\mathbf{0}
$$


where $\mathbf{M}$ is a $4 \times 8$ matrix whose entries are dependent on $\tan (\theta)$ and the invariants $K$ 's and $J$ 's,

$$
\mathbf{X}=\left[\begin{array}{llllll}
X_{12} & Y_{12} & X_{13} & Y_{13} & X_{14} & Y_{14}
\end{array}\right]^{t} .
$$

If the camera undergoes rotation with another angle $\theta^{\prime}$, and we know the matching points of the first view, then we have a relationship similar to Equation 14

$$
\mathbf{N}\left(\theta^{\prime}\right) \mathbf{X}=\mathbf{0}
$$

Putting Equation 14 and Equation 16 together we obtain

$$
\mathbf{A}\left(\theta, \theta^{\prime}\right) \mathbf{X}=\mathbf{0}, \mathbf{A}\left(\theta, \theta^{\prime}\right)=\left[\begin{array}{l}
\mathbf{M}(\theta) \\
\mathbf{N}\left(\theta^{\prime}\right)
\end{array}\right]
$$

Equation 17 is a set of 8 nonlinear equations in $\mathbf{X}$, $\tan (\theta)$ and $\tan \left(\theta^{\prime}\right)$. Because $\mathbf{X}$ is nonzero vector, we have that $\operatorname{det}\left(\mathbf{A}^{T} \mathbf{A}\right)=0$, which is a polynomial equation in $\tan (\theta)$ and $\tan \left(\theta^{\prime}\right)$. Given a reference view with $\theta^{\prime}=\theta_{0}$, e.g., $\theta^{\prime}=0$, which we can take as the orientation initialization of the $3 \mathrm{D}$ world coordinate system, we can easily get any other rotation angle $\theta$ by solving the polynomial equation. If the initial orientation is not set, the rotation angle can only be determined up to a shift of any given angle.

Moreover, $\mathbf{X}$ is the eigenvector of the smallest eigenvalue of $\mathbf{A}^{T} \mathbf{A}$ and the Euclidean coordinates can be determined up to a scale assuming the first point to be the origin. The scale can be set by knowing any one length.

\subsection{Planar motion and rotation about $\mathrm{Y}$ axis}

Let $\mathbf{X}_{w} \mathbf{Y}_{w} \mathbf{Z}_{w}$ be the world coordinate system. Consider restricted motion with rotation $\theta$ only about the $\mathbf{Y}_{w}$-axis. This would be the case of a robot moving on the ground with a camera mounted horizontally (we look normally, at last). Consider three points in the world coordinate system $\left(X_{i}, Y_{i}, Z_{i}, 1\right),\left(X_{j}, Y_{j}, Z_{j}, 1\right)$ and $\left(X_{k}, Y_{k}, Z_{k}, 1\right)$, with the coordinates of their images being $\left(u_{i}, v_{i}, 1\right),\left(u_{j}, v_{j}, 1\right)$ and $\left(u_{k}, v_{k}, 1\right)$, respectively.

By projecting the $3 \mathrm{D}$ control points on to the image plane, we have

$$
\begin{aligned}
& u_{p}=f_{x} \frac{X_{p} \cos \theta+Z_{p} \sin \theta+t_{1}}{-X_{p} \sin \theta+Z_{p} \cos \theta+t_{3}}+u_{0} \\
& v_{p}=f_{y} \frac{Y_{p}+t_{2}}{-X_{p} \sin \theta+Z_{p} \cos \theta+t_{3}}+v_{0}
\end{aligned}, p \in\{i, j, k\} .
$$

Now, let the first two points $i$ and $j$ be on the $\mathbf{Y}_{w}$ axis and the third point $k$ be on the $\mathbf{X}_{w} \mathbf{Y}_{w}$ plane. By eliminating the internal parameters of the camera from the above equation we obtain

$$
\begin{aligned}
& K_{k}=\frac{u_{i}-u_{j}}{u_{i}-u_{k}}=0 \\
& J_{k}=\frac{v_{i}-v_{j}}{v_{i}-v_{k}}=\frac{Y_{i}-Y_{j}}{\left(Y_{i}+t_{2}\right)-\frac{Y_{k}+t_{2}}{1-X_{k} \frac{s_{i n t}}{t_{3}}}} .
\end{aligned}
$$

If we consider another point $l$ on the $\mathbf{X}_{w} \mathbf{Y}_{w}$ plane, we obtain, similarly,

$$
\begin{aligned}
& K_{l}=\frac{u_{i}-u_{i}}{u_{i}-u_{l}}=0 \\
& J_{l}=\frac{v_{i}-v_{i}}{v_{i}-v_{l}}=\frac{Y_{i}-Y_{j}}{\left(Y_{i}+t_{2}\right)-\frac{Y_{i}+t_{2}}{1-X_{l} \frac{\operatorname{sin\theta }}{t_{3}}}} .
\end{aligned}
$$

Because $K_{k}$ and $K_{l}$ vanish, we can separate the terms of $\sin \theta$ and $t_{3}$ to obtain

$$
\begin{aligned}
& \frac{t_{3}}{\sin \theta}=\frac{X_{k}\left(\left(1-J_{k}\right) Y_{i}-Y_{j}-J_{k} t_{2}\right)}{Y_{i}-Y_{j}+J_{k}\left(Y_{k}-Y_{i}\right)} \\
& =\frac{X_{l}\left(\left(1-J_{l}\right) Y_{i}-Y_{j}-J_{l} t_{2}\right)}{Y_{i}-Y_{j}+J_{l}\left(Y_{l}-Y_{i}\right)}=T_{s} .
\end{aligned}
$$

$t_{2}$ can be easily found from Equation 21. In order to find $\theta$, we have to know another control point lying on the plane $\mathbf{Y}_{w} \mathbf{Z}_{w}$, say $m$. Following exactly the derivation process of Equation 21, we can obtain a similar relationship as follows

$$
\frac{t_{3}}{\cos \theta}=-\frac{Z_{m}\left(\left(1-J_{m}\right) Y_{i}-Y_{j}-J_{m} t_{2}\right)}{Y_{i}-Y_{j}+J_{m}\left(Y_{m}-Y_{i}\right)}=T_{c} .
$$

Since we know $t_{2}, T_{s}$ and $T_{c}$ could be computed, then $\tan \theta=T_{c} / T_{s}$ and $t_{3}=T_{s} \sin \theta=T_{c} \cos \theta$.

In Equation $18, K_{k}$ vanishes because both $3 \mathrm{D}$ points $i$ and $j$ are on the $\mathbf{Y}_{w}$ axis. $K_{k}$ would not be zero if we choose 3 points in general position. Now that we know $\theta, t_{2}$ and $t_{3}$, solving for $t_{1}$ is trivial. If three points $\left(X_{i}, Y_{i}, Z_{i}, 1\right),\left(X_{j}, Y_{j}, Z_{j}, 1\right)$ and $\left(X_{k}, Y_{k}, Z_{k}, 1\right)$ are in general position, then the image measurement $K_{i j k}$ does not vanish and we have

$$
t_{1}=\frac{K_{i j k}\left(\frac{b}{a}-\frac{f}{e}\right)-\left(\frac{b}{a}-\frac{d}{c}\right)}{\left(\frac{1}{a}-\frac{1}{c}\right)-K_{i j k}\left(\frac{1}{a}-\frac{1}{e}\right)},
$$

where $a=-X_{i} \sin \theta+Z_{i} \cos \theta+t_{3} ; b=X_{i} \cos \theta+Z_{i} \sin \theta$; $c=-X_{j} \sin \theta+Z_{j} \cos \theta+t_{3} ; d=X_{j} \cos \theta+Z_{j} \sin \theta ;$ $e=-X_{k} \sin \theta+Z_{k} \cos \theta+t_{3}$; and $f=X_{k} \cos \theta+Z_{k} \sin \theta$.

Thus, in this special case, we obtain a closed form solution to the localization problems with specially located landmarks - two on the $\mathbf{Y}_{w}$ axis, two on the $\mathbf{X}_{w} \mathbf{Y}_{w}$ plane and one on the $\mathbf{Z}_{w} \mathbf{Y}_{w}$ plane.

\section{Results}

We present some results of both simulations and tests carried out on a experimental set up.

In Figure ??, we show images of two positions of a calibration object obtained using a stationary Pulnix CCD camera. The world coordinates corresponding to both the positions of the calibration object are known and they serve as Euclidean landmarks. We refer to the world points (corners) on our calibration objects with numbers assigned according to a row major order. 
In Table ?? we give the camera external parameters for four stations of the camera as obtained using Tsai's calibration method [15]. We assume that all correspondence information is known (hand picked).

In Table ??, we plot the differences between some three point invariants as measured from the image and as obtained synthetically by projecting the world coordinates according to Equation ??. We find that the actual measurements adhere to the projection model with reasonable accuracy.

\section{Conclusion}

We have described image measurements which are camera invariant but location variant. Using such uncalibrated image measurements we have presented a method of estimation of camera externals from known Euclidean landmarks. We have also shown that it is possible, using the uncalibrated image invariants, to compute the Euclidean structure of world points from multiple views with known camera positions. Our initial experiments show that the invariants are stable and it is indeed possible to compute the position information reliably.

The landmark based self-localization presented in this paper does not require camera calibration making it suitable for robot navigation applications where accurate calibration is not possible due to continually changing parameters and parts of the system. The method is also ideally suited for visual servoing using a feed-back loop.

The method can capitalize on the benefits of both image and landmark-based methods. Images can be encoded as a set of visual features called landmarks. Potential landmarks can be detected using an attention mechanism implemented as a measure of uniqueness. These can then be selected and represented by an appearance-based encoding. Localization can be performed using a landmark tracking and interpolation method which obtains an estimate accurate to a fraction of the environment sampling density. Localization can thus be a two-step process consisting of an off-line preprocessing stage and an on-line estimation stage. The off-line stage may consist of building a representation of the environment in the form of an invariants database, which is later used for positioning. The online stage can then use the database to match currently observed invariants to previously stored invariants using an interpolation method. These matches can then used to compute individual position estimates in a robust fashion.

We believe that that the camera invariant measurements described in this paper can be used in a variety of robotic applications.

\section{References}

[1] D. Avis and H. Imai, "Locating a robot with angle measurements", Journal of Symbolic Computation, no. 10, pp. 311-326, 1990.

[2] R. Basri and E. Rivlin, "Localization and homing using combinations of model views", Artificial Intelligence, vol. 78, no. 1-2, pp. 327-354, October 1995 .

[3] G. Dudek and C. Zhang, "Vision-based robot localization without explicit object models", in Proc. IEEE Int. Conf. on Robotics and Automation, 1996.

[4] John W. Eaton, Octave - A high-level interactive language for numerical computations, Version 1.1 .1

[5] O. Faugeras, "Three-Dimensional Computer Vision: A Geometric Viewpoint", The MIT Press, 1996.

[6] O. Faugeras, L. Quan and P. Strum, "Selfcalibration of a 1D Projective Camera and its Application to the Self-Calibration of a 2D Projective Camera", in Proc. ECCV'98.

[7] E. Krotkov, "Mobile robot localization using a single image", in Proc. 1989 IEEE Int. Conf. on Robotics and Automation, pp. 978-983. 1989.

[8] J. J. Leonard and H. F. Durrant-Whyte, "Mobile robot localization by tracking geometric beacons", IEEE Transactions on Robotics and Automation, vol. 7, no. 3, pp. 376-382, 1991.

[9] S.K. Nayar, H. Murase, and S.A. Nene, "Learning, positioning, and tracking visual appearance", in Proc. IEEE Conf on Robotics and Automation, San Diego, CA, May 1994, pp. 3237-3246.

[10] L. Quan and Z. Lan, "Linear $N \geq 4$ Point Pose Determination", in Proc. ICCV'98, pp. 778-783.

[11] R. Sim and G. Dudek, "Mobile Robot Localization from Learned Landmarks", Proc. IEEE/RSJ Conf. on Intelligent Robots and Systems (IROS), Victoria, BC, Oct. 1998.

[12] K. Sugihara, "Some location problems for robot navigation using a single camera", Computer Vision, Graphics, and Image Processing, vol. 42, pp. 112-129, 1988.

[13] K. T. Sutherland and W. B. Thompson, "Pursuing projections: Keeping a robot on path", in 
Proc. IEEE Int. Conf. on Robotics and Automation, San Diego, CA, May 1994, pp. 3355-3361, IEEE Computer Society Press.

[14] S. Thrun, "Finding landmarks for mobile robot navigation", in Proc. IEEE Int. Conf. on Robotics and Automation, Leuven, Belgium, May 1998, pp. $958-963$.

[15] Roger Y. Tsai, "A versatile Camera Calibration Technique for High-Accuracy 3D Machine Vision Metrology Using Off-the-Shelf TV Cameras and Lenses", IEEE Journal of Robotics and Automation, Vol. RA-3, No. 4, August 1987, pp. 323-344.

\section{Appendix}

When the internal parameters change across views, 2 views are not sufficient to reconstruct the $3 \mathrm{D}$ scene. Because the $2 \mathrm{D}$ camera can be viewed as 2 separate $1 \mathrm{D}$ camera, we prove this observation by use of $1 \mathrm{D}$ camera.

Suppose we have $N$ views taken by $1 \mathrm{D}$ camera with the internal parameters of the camera changing across views, the number of unknown internal parameters is $2 N$. The number of $3 \mathrm{D}$ pionts to be reconstructed on the trifocal plane is $n$, therefore we have $2 n$ variables of $3 \mathrm{D}$ entities. While we have totally $N n$ equations to solve this problems. In order for this problem to be sovable, the relationship $N n \geq 2(N+n)$ must be satisfied. When $N=2$, the above relationship never establish.

As a byproduct of this observation, we are not able to interpolate the third view/position in the cases of 2D/3D interpolation, i.e., at least four views/stations are required. 